\title{
Presencia de Emilia Pardo Bazán en las bibliotecas virtuales y análisis de las versiones digitales de sus obras
}

\author{
Pedro Incio \\ Universidade da Coruña \\ pincio@udc.es
}

(recibido decembro/2016, revisado decembro/2016)

RESUMEN: El objetivo de este trabajo es analizar la presencia de Emilia Pardo Bazán (EPB) en las bibliotecas virtuales y evaluar las versiones digitales de las obras presentes en ellas. Para ello se han revisado diferentes colecciones de las principales bibliotecas digitales con fondos de Doña Emilia. Estas han sido la biblioteca de la Real Academia Galega (RAG), Galiciana (Gal.), Hispana (Hisp.) y Europeana (Eur.), la Biblioteca Digital Hispana de la Biblioteca Nacional de España (BDH) y la Biblioteca Virtual Miguel de Cervantes (BVMC). Con todo ello se ha elaborado un listado de las ediciones digitales de sus obras. Las obras presentes en Hispana no se han utilizado pues todas están también presentes en Galiciana o en la BDH.

Las búsquedas se han hecho tomando como punto de partida la bibliografía aparecida en 1981 en la tesis de Nelly Clemessy: Emilia Pardo Bazán como novelista, a la que se han añadido los títulos presentes con ediciones digitales en la colección de la RAG; por último, se ha cotejado con las primeras Obras completas de EPB.

Posteriormente se han evaluado las diferentes versiones y prestaciones de las obras digitales y de las páginas web que las alojan, con el fin de ayudar al investigador a dirigirse a las ediciones más autorizadas.

PALABRAS CLAVE: Emilia Pardo Bazán, Bibliotecas virtuales, Libros electrónicos, Obras completas, Digitalización.

ABSTRACT: The objective of this work is to analyze the presence of Emilia Pardo Bazán (EPB) in the virtual libraries and to evaluate the digital versions of the works present in them. For this, different collections of the main digital libraries with funds from Doña Emilia have been reviewed. These have been the library of the Galician Royal Academy (RAG), Galiciana (Gal.), Hispana (Hisp.) And Europeana (Eur.), Hispanic Digital Library of the National Library of Spain (BDH) and the Miguel de Cervantes Digital Library (BVMC). With all this a list of the digital editions of his works has been elaborated. The works present in Hispana have not been used since all are also present in Galiciana or in the BDH.

The researches have been made taking as a starting point the bibliography appeared in 1981 in the thesis of Nelly Clemessy: "Emilia Pardo Bazán como novelista", to which the present titles with digital editions have been added in the collection of the RAG; Finally, has been compared with the first complete Works of EPB.

Subsequently the different versions and features of the digital works and the web pages that host them have been evaluated, in order to help the researcher to address the most authoritative editions.

KEY WORDS: Emilia Pardo Bazán, Virtual libraries, Electronic books, Complete works, Digitalization.

La bibliografía de y sobre Emilia Pardo Bazán es ingente. En noviembre de 2016, sólo en la Biblioteca Nacional de España encontramos 509 registros de autoría y 134 estudios sobre EPB; la Biblioteca de la Universidade de Santiago, por su parte nos muestra 818 registros bajo la entrada "Pardo Bazán, Emilia, Condesa de, 1851-1921" así como 1.264 entradas bajo la materia "Pardo Bazán, Emilia, Condesa de, 1851 1921". En Worldcat, el catálogo colectivo más grande del mundo, aparecen 1.196 referencias, de ellas 1.126 
son referencias de autoría y 187 se corresponden con libros digitales. Por ello este trabajo se limitará a la bibliografía histórica tomando como base la aparecida en la tesis doctoral de Nelly Clemessy. En esta monografía aparece la obra de EPB con los títulos de sus principales ediciones por el orden cronológico de su primera publicación, y además nos ofrece un corto listado de ediciones no fechadas. Esta bibliografía inicial se ha ampliado con los datos que nos ofrece el catálogo de la Real Academia Galega. La tarea de elaborar una bibliografía de EPB está pendiente y nos proponemos elaborarla más adelante. ${ }^{1}$

Quedan fuera muchas obras de EPB, pero el objetivo de este estudio es valorar las ediciones digitales de EPB y por ello nos interesan las ediciones históricas, aquellas que la autora llevó a la imprenta, y en su primera salida. Se han estudiado sólo sus obras principales y se han dejado para otra ocasión los otros tipos de materiales de una autora tan prolífica, como son sus numerosos discursos, los títulos recopilatorios o los muchos folletos; $y$, por supuesto sus abundantísimas colaboraciones en publicaciones seriadas.

\section{EUROPEANA}

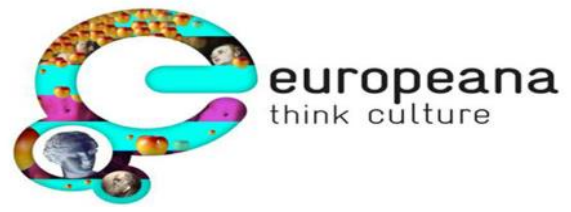

Europeana es la gran biblioteca digital europea, con registros de las distintas bibliotecas nacionales y de otros importantes centros. Nos da acceso a casi 54 millones de documentos; entre los que tenemos 30 millones de imágenes, 22 millones de textos y dos millones de otros tipos de material; entre los países proveedores aparece España con cinco millones de documentos.

De ese total de 54 millones de documentos, 17 millones aparecen con enlace a los objetos digitales, es decir que dan acceso a los propios documentos, y también nos informan de los permisos para su uso, mientras que los otros son tan sólo referencias bibliográficas. Entre los 17 millones de objetos podemos separar las diferentes tipologías: Imagen $(12,304,160)$, Texto $(4,024,092)$, Video $(126,134)$ Sonido $(47,426) 3 D(3,151)$.

La búsqueda "Emilia Pardo Bazán" en Eur. nos ofrece 60.000 resultados, algo inoperativo, pero desde cada registro bibliográfico podemos afinar la búsqueda, mediante la opción de enlazar con el campo Creador. Así, la búsqueda "Emilia Pardo Bazán" como Creador nos da acceso a 405 referencias, localizadas en las siguientes instituciones: Biblioteca Virtual Miguel de Cervantes (219), Real Academia Galega (67), Galiciana (39), National Library of Spain (36), Bodleian Libraries, University of Oxford (34), Complutense University Library of Madrid (3), Biblioteca Virtual del Patrimonio Bibliográfico (1) y Minerva (Universidade de Santiago de Compostela, Spain) (1).

En la búsqueda por Instituciones, los registros nos envían a las obras digitales en las ediciones correspondientes. La búsqueda en Europeana sirve para encontrar en una sola búsqueda los registros, pero es un mero localizador y las obras están en las páginas respectivas; por lo que no se puede estudiar la obra digital más que en el sitio de origen.

La búsqueda nos ofrece registros bibliográficos muy completos y con abundantes enlaces. Al lado de los registros aparece la imagen de la cubierta de la obra que nos permite enlazar con la edición digital. Estas ediciones están localizadas en las bibliotecas que han digitalizado la obra. El uso de Europeana no es muy recomendable debido a que al ser una base de datos que se nutre de las aportaciones de otras bases de datos, las búsquedas ofrecen mucho ruido documental e inconsistencias. Por ello es necesario trabajar con las distintas instituciones que envían sus registros a Europeana, como en nuestro caso haremos con Hispana y Galiciana, la BDMC y la BDH.

\footnotetext{
${ }^{1}$ Una aproximación a la bibliografía de EPB, con los títulos presentes en el catálogo de la Biblioteca Nacional de España en: http://www.refworks.com/refshare2?site=041191162267200000/RWWEB105509443/LA\%20TRIBUNA\%203
} 
HISPANA

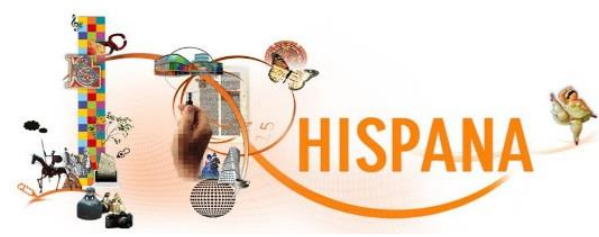

Hispana es el portal de acceso a la cultura digital española y el agregador nacional de contenidos a Europeana. Da acceso a siete millones de objetos digitales pertenecientes a 208 repositorios, y sus objetos están integrados en Europeana. Es importante señalar que para participar los repositorios han de cumplir los protocolos europeos de metadatos.

La página de Inicio de Hispana nos ofrece ya una caja de búsqueda, pero es más operativo utilizar la pestaña de Búsqueda de esa misma página principal. En esta página de búsqueda podemos hacer uso de varios campos: Cualquier campo, Título, Autor, Tema y Descripción, Año de publicación y Colección - con un listado de las instituciones que participan.

La búsqueda de Autor con "Pardo Bazán, Emilia 1851-1921" nos da un listado con 155 registros de diferentes procedencias: Biblioteca digital de la Real Academia Galega (48) Biblioteca Digital Hispánica (41) Galiciana (35) Hemeroteca Virtual de la Real Academia Galega (19), y también aparecen otras bibliotecas con pocos registros. Todas las búsquedas son lanzadas a la vez en Hispana y Europeana, y por ello nos presenta también un enlace a sus resultados; lo cual permite navegar de una a otra colección. Es importante señalar que 31 de los resultados de Galiciana se corresponden con los números publicados del Nuevo Teatro Crítico, así como con las aportaciones en publicaciones seriadas de la Hemeroteca Virtual da Real Academia Galega.

Las búsquedas nos permiten filtrar por procedencia y así recoger los objetos de una institución, como por ejemplo los 48 títulos de la RAG, y a partir de estos registros bibliográficos, acceder al texto completo del libro en su versión de la RAG. La mayoría de los libros presentes en Hispana pertenecen a la BDH, Gal. y la RAG. No forma parte del proyecto la BVMC.

Hispana no tiene control de autoridades y por ello no existen enlaces desde los campos de autoría a las obras. Los registros bibliográficos del listado nos envían a las ediciones digitales en las bibliotecas correspondientes. El enlace al libro aparece en el campo Identificador del recurso y nos envía al ejemplar de la biblioteca digital correspondiente. Por ello, al igual que la biblioteca anterior, su uso es válido para encontrar los títulos, pero no para revisar las ediciones.

\section{GALICIANA}

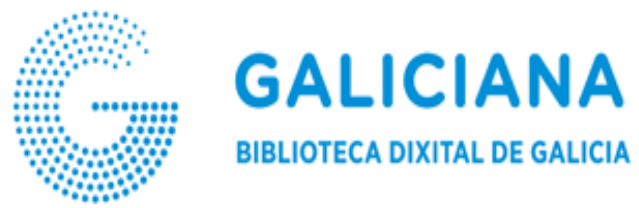

Galiciana es la biblioteca digital gallega impulsada por la Xunta de Galicia. El proyecto tiene como objetivo no solo la digitalización de los fondos patrimoniales más relevantes para Galicia, sino también la normalización del acceso mediante la aplicación de las últimas directrices y estándares nacionales e internacionales, incluyendo los referidos a los metadatos. Su finalidad es lograr la máxima visibilidad de los recursos digitales integrados en ella, así como la interoperabilidad con los principales proyectos de digitalización existentes hoy en día. Desde sus inicios Galiciana ha estado presente en Hispana, directorio y recolector de recursos digitales españoles, y en Europeana, la biblioteca digital europea surgida como una forma de contribuir a la preservación y difusión de la riqueza cultural de Europa.

La colección consta de5.650 títulos, de los que 4.600 son libros. La RAG es una de las instituciones con más aportaciones, pues en las estadísticas de Gal. aparecen 805 libros de la sede coruñesa, y sólo es superada en número por la propia Biblioteca de Galicia y la Universidade compostelana.

La página principal tiene una pestaña de Búsqueda y otra de Búsqueda avanzada, además de permitirnos trabajar con otros campos: Hemeroteca, Títulos, Autores, Lugares -de edición-, Editores y Materias. 
Desde la página de Búsqueda se nos permite utilizar distintos campos, y en la caja de los mismos se nos muestran las distintas opciones al teclear nuestras palabras clave. Así la Búsqueda de Autor nos permite seleccionar "Pardo Bazán, Emilia, Condesa de, 1851-1921". Gal. nos permite buscar en el texto completo, puesto que todas las páginas de Galiciana han sido transformadas por un programa de OCR. La opción de texto completo incrementa mucho los resultados, aunque la utilidad de los mismos es relativa para la mayoría de los estudios; por ejemplo, al utilizar "pardo bazan Emilia" recuperamos 3.581 registros, pero de ellos la mayoría, 3.452, pertenecen a las colecciones hemerográficas.

Galiciana sí tiene control de autoridades, de forma que desde cualquier registro bibliográfico de EPB la entrada de Autor "Pardo Bazán, Emilia, Condesa de, 1851-1921" nos permite acceder al registro de autoridad. Este nos envía al VIAF, Virtual Internet Authority File, la base de datos mundial de nombres de autores, y en ella aparecen múltiples referencias de véase. Este control de las formas de los nombres, esencial para evitar duplicados, es muy importante y se basa en un trabajo minucioso que es necesario que abarque a todos los autores, algo que de momento no se ha hecho. El resultado de esta búsqueda son 62 obras en Gal. Y además se nos enlaza con los 48 resultados en Europeana, con lo que podemos enlazar con sus títulos o los de sus componentes, como la BDH o la BVMC.

Los registros aparecen ordenados por título, pero se nos permite reordenarlos por Autor, Fecha de Publicación y Relevancia. Tanto desde el listado de registros como desde cada uno de ellos podemos enlazar a la edición digital del título. En el caso de que un título tenga versiones en distintas bibliotecas, el campo Ejemplares nos permite elegir una de ellas y comparar las distintas copias.

La edición digital del título nos muestra en cada pantalla 12 páginas de la obra; esto nos permite ver en un pantallazo la cubierta, la portada y otros preliminares, por ejemplo. Clicando en cada imagen accedemos a cada una de las páginas de la obra. La opción Desargar/lmprimir nos permite entonces seleccionar el formato entre PDF, versión imprimible, e Imagen JPG, versión descargable. Con la versión en pdf podemos descargar o imprimir el documento entero o una selección de páginas, mientras que la versión jpg nos obliga a imprimir página a página. Gal. permite ejecutar las búsquedas en el texto completo, también unifica las distintas ediciones y permite la búsqueda de las mismas

\section{BIBLIOTECA DIGITAL HISPANA}

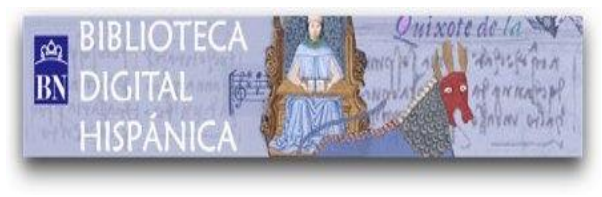

La Biblioteca Digital Hispánica es la biblioteca digital de la Biblioteca Nacional de España. Proporciona acceso libre y gratuito a más de 180 mil documentos digitalizados, entre los que se cuentan libros impresos entre los siglos XV y XIX, manuscritos, dibujos, grabados, folletos, carteles, fotografías, mapas, atlas, partituras, prensa histórica y grabaciones sonoras.

Hoy en día incorpora más de 180.100 títulos, de todas las temáticas y tipos documentales: Monografías impresas, 72.584; Manuscritos, 13.477; Dibujos, Grabados y Fotografías, 35.619; Partituras, 31.266; Material cartográfico, 6.894; Registros sonoros, 20.122; Prensa y revistas, 1.161. Una opción interesante es la de filtrar por colecciones destacadas, donde encontramos, entre otras muchas colecciones: Cervantes (2426), Atlas (2063), Incunables (1417), Carteles de la Guerra Civil (450), Ephemera (410), Obras de Goya, (210), Grabados de Durero (203). Como curiosidad, sorprende que el autor con más documentos en la BDH sea Verdi, seguido del fotógrafo J. Laurent y con Cervantes en el tercer puesto.

Al igual que Gal., la página principal nos muestra una caja de Búsqueda, aunque también aparece una opción de Busque en el texto de los documentos y otra de Búsqueda avanzada. La búsqueda simple sólo usa los campos básicos mientras que la avanzada nos permite utilizar todos los campos o alguno de ellos, y nos permite también marcar un intervalo de fechas o elegir entre muchos tipos de documentos y lenguas.

La búsqueda de Autor: "Pardo Bazán, Emilia, Condesa de (1851-1921)" nos recupera 44 registros. El listado de títulos se presenta ordenado por relevancia, pero también se puede reordenar por título, autor, 
fecha. Se nos permite filtrar los resultados por Acceso temático, Tipo de material, Colecciones destacadas, Autor, Lengua y Año.

El listado de títulos recuperados ofrece muchas opciones novedosas, todas en la línea de la llamada web 2.0, por ejemplo, nos permite compartir los datos de cada libro en las distintas redes sociales, así como conseguir la url permanente del documento, también deja hacer comentarios sobre los títulos, lo cual es una opción interesante. Aparece también una opción novedosa: escuchar el texto, si bien hay que decir que muchos textos no presentan esta utilidad y en los casos en que se puede escuchar un texto es un robot el que lee más mal que bien la obra.

Cada uno de los libros nos muestra un registro bibliográfico sencillo con la opción de enlazar al catálogo de la Biblioteca Nacional, y también la cubierta de la obra. Otra opción interesante es la de "Otros usuarios han visto", donde, al igual que muchas plataformas comerciales, se nos da noticia de los libros que han revisado otros usuarios de la $\mathrm{BNH}$, de los cuales se entiende que pueden coincidir con nuestros intereses.

La página permite las descargas en pdf, jpg y txt. De este último formato la página nos avisa de que el texto se obtiene a partir de un proceso de OCR y por ello su calidad puede variar en función de la tipografía original del documento.

El listado de títulos es muy interesante pues algunos, sobre todo del siglo XX, no aparecen en las otras bibliotecas digitales.

\section{REAL ACADEMIA GALEGA}

\section{REAL ACADEMIA GALEGA}

La RAG cuenta con varias bibliotecas personales que ha podido reunir a lo largo de su historia, entre ellas la de EPB, y entre su colección privada encontramos la mayoría de sus propias obras.

La Biblioteca de la RAG dispone de una biblioteca digital denominada "Monografías séc. XIX." y en ella tenemos cerca de 800 obras, con más de 120.000 páginas, y entre éstas encontramos los títulos de EPB. En el catálogo de la biblioteca la búsqueda de Autor "Pardo Bazán, Emilia, Condesa de", nos ofrece 220 títulos, de los que 52 están digitalizados en la sección dedicada a las monografías del siglo XIX. El catálogo de la biblioteca no enlaza con las versiones digitales de la sección la llamada Monografías S.XIX, por ello debemos hacer la búsqueda en la sección digitalizada. Las búsquedas en esta sección solo pueden ejecutar con los campos de autor, título y año.

La búsqueda de EPB en la colección digital nos presenta un listado de obras desde las que podemos enlazar con la versión jpg del libro. Los libros digitales están en formato jpg; sólo podemos imprimir hoja a hoja y no se puede descargar el archivo. Por contra, las mismas copias en Gal. nos permiten descargar e imprimir en pdf y jpg. Es entonces más recomendable el uso de Gal. que el de la RAG. Debido a que las obras no han pasado por un proceso de OCR, en la RAG no podemos hacer búsquedas en el texto completo, lo cual, si bien para las búsquedas generales puede ser poco operativo, para trabajos sobre un título específico nos brinda muchas oportunidades.

Es importante resaltar que muchas obras pertenecen a la primera salida de sus Obras completas y por ello no aparecen las primeras ediciones de los títulos de Doña Emilia.

\section{BIBLIOTECA VIRTUAL MIGUEL DE CERVANTES}

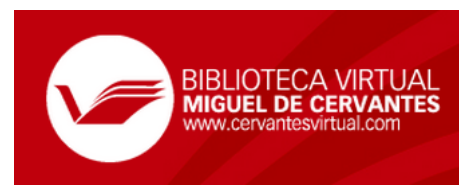

La Fundación Biblioteca Virtual Miguel de Cervantes gestiona la Biblioteca Virtual Miguel de Cervantes. La BVMC inició su desarrollo en 1998 y se presentó un año después como un fondo virtual de obras clásicas 
en lenguas hispánicas con un sistema de ordenación y búsqueda similar a una biblioteca y acceso gratuito a través de internet.

La Biblioteca Virtual Miguel de Cervantes ofrece en estos momentos más de 550 portales dedicados a autores fundamentales del español, también a instituciones y a variados núcleos temáticos, además de contar un fondo que supera los 195.000 registros bibliográficos.

Al igual que las otras bibliotecas virtuales, la página principal nos muestra una caja de búsqueda, pero también una pestaña de búsqueda avanzada. Esta búsqueda avanzada nos ofrece dos opciones, la búsqueda en el catálogo y la búsqueda en el contenido, la cual viene a ser la búsqueda en el texto completo, de la cual ya hemos hablado. La búsqueda en el catálogo nos permite filtrar por tipo de documento mientras que el contenido podemos restringirlo a un título o a un autor; además nos permite seleccionar los documentos entre HTML, PDF o Portales.

La búsqueda de Autor "Pardo Bazán, Emilia, Condesa de, 1851-1921", nos ofrece 362 registros que pueden ordenarse por título o autor. El programa nos muestra las fichas de los títulos con un icono que nos da acceso a la obra en html, acompañado de otro que permite buscar en el texto completo de esta obra; algunos registros están en pdf y no permiten búsquedas. También tenemos un abundante fondo de registros sonoros que nos permiten escuchar la obra.

La búsqueda por la materia "Pardo Bazán, Emilia, Condesa de, 1851-1921" recupera 393 obras que se pueden ordenar por título o autor. Los registros bibliográficos nos permiten enlazar con los portales temáticos a los que se adscribe el título. El icono html de cada registro nos da acceso a la obra mientas que la opción Concordancias nos permite buscar las palabras que aparecen en la obra. Los registros en html nos llevan a la edición web de la obra, que aparece introducida por una nota con los datos de la edición original. La opción Colección nos lleva a partes de obras; también podemos ir desde una obra a su documento fuente, p. e. de un cuento a la recopilación en que ha aparecido. En el caso de Los pazos de Ulloa, por ejemplo, el registro nos lleva a los 30 audios de los distintos capítulos de la novela. En el caso de las publicaciones periódicas, el registro bibliográfico nos lleva a los registros de sus partes, como es el caso del Nuevo Teatro Crítico, que enlaza con los 30 números disponibles. En lo que atañe a EPB es de resaltar que aparecen muchas colaboraciones periodísticas, en Blanco y Negro, El Imparcial, Nuevo Teatro Crítico, etc.

La BVMC incorpora algunas opciones novedosas, como que el registro bibliográfico nos permite citar la obra en los formatos RIF y CIS, o la opción web semántica, que nos permite encontrar obras relacionadas, cargar los metadatos, buscar en otras páginas o compartir la obra en las redes sociales

Desde el campo Portales del registro bibliográfico podemos acceder a información ampliada sobre los autores. La web tiene secciones dedicadas a los grandes escritores y en el caso de EPB es ciertamente muy completa y de gran calidad. Así tenemos: Presentación, La autora -biografía y cronología-, Su obra -el catálogo de la BVMC y bibliografía-, Estudios e investigación, Fonoteca, Imágenes -Album de fotos, Video y televisión, Videoteca- y Enlaces.

La colección de EPB es la más completa de las que hemos revisado y la digitalización en html permite muchas opciones de trabajo. Sin embargo, muchas de sus versiones están hechas a partir de ediciones tardías de las obras, como las célebres de Aguilar de los 60 o las muy populares de Espasa en los 80, y por ello apenas tienen interés filológico; las primeras ediciones de EPB aparecen en las bibliotecas virtuales ya revisadas. 


\section{ANEXO}

Se presenta un listado con las obras de EPB ordenadas cronológicamente. La bibliografía se basa en la que publicó Nelly Clemessy en 1981, aunque se han fechado las obras que aparecían allí sin datar, un trabajo posible gracias al acceso virtual a los diferentes ejemplares. A esta bibliografía se han añadido las obras que, en versión digital, están presentes en la colección de la RAG. Por último, se ha cotejado con las primeras Obras completas de EPB, publicadas a lo largo de su vida por varias casas editoras.

Las obras aparecen en un listado con la fecha de su primera edición. Las localizaciones de los distintos títulos digitalizados aparecen con la abreviatura de la biblioteca en la que se encuentran (RAG, Gal., BDH, BVMC); además, si la fecha de la edición del ejemplar digitalizado no coincide con la fecha de aparición del libro, se pone esa segunda fecha al lado de la abreviatura.

Por último, aparecen en negrita los títulos que no se han encontrado en las bibliotecas revisadas. Como excepción aparece la obra De siglo a siglo, 1902, digitalizada por la Bodleian Library. También se han revisado las colecciones analógicas de la biblioteca de la RAG y se han encontrado ejemplares de casi todos los títulos que habría que digitalizar para completar la edición digital de sus obras completas. Los títulos que aparecen en el catálogo de la RAG, pero que aún no han sido digitalizados, son las siguientes: Cuentos de Navidad y de Reyes, Goya y la espontaneidad española, Cada uno, Allende la verdad, Finafrol, En las cavernas, Cocina española antigua, Cocina española moderna, Porvenir de la literatura después de la guerra, Clavileño, Rodando. Se han marcado en cursiva. 


\begin{tabular}{|c|c|c|}
\hline Título de la obra & Año de edición & Versiones digitales \\
\hline Pascual López & 1879 & RAG, Gal., BVMC 1996 \\
\hline El Príncipe Amado & 1879 & RAG, Gal., BVMC \\
\hline Un viaje de novios & 1881 & RAG, Gal., BDH, BVMC \\
\hline Jaime (poema) & 1881 & RAG, Gal., BDH 1886, BVMC \\
\hline San Francisco de Asís (siglo XIII) & 1882 & RAG, Gal.) \\
\hline La Tribuna & 1883 & RAG 1893? Gal. 1882? \\
\hline La Cuestión palpitante & 1883 & RAG 1891, Gal.1891, BVMC, BDH \\
\hline $\begin{array}{l}\text { Bases del Folk-lore Español. Y reglamento } \\
\text { del Folk-lore Gallego }\end{array}$ & 1884 & Gal. \\
\hline El cisne de Vilamorta & 1885 & RAG, Gal., BDH, BVMC \\
\hline El folclore gallego en 1885-1885 & 1886 & RAG, Gal., BDH, BVMC \\
\hline Los Pazos de Ulloa & 1886 & RAG, Gal., BDH, BVMC \\
\hline La madre naturaleza & 1887 & RAG, Gal., BDH, BVMC \\
\hline La revolución y la novela en Rusia & 1887 & RAG, Gal. \\
\hline La Leyenda de la Pastoriza & 1887 & RAG, Gal. \\
\hline De mi tierra & 1888 & RAG, Gal. \\
\hline Mi romería & 1888 & RAG, Gal., BDH \\
\hline Los pedagogos del Renacimiento & 1889 & RAG, Gal. \\
\hline Insolación & 1889 & RAG, Gal., BVMC \\
\hline Morriña (historia amorosa) & 1889 & RAG, Gal., BDH, BVMC \\
\hline Al pie de la torre Eiffel & 1889 & RAG, Gal., BDH, BVMC 1899 \\
\hline Un Destripador de antaño & [189-?] & RAG, Gal., BDH 1890 \\
\hline $\begin{array}{l}\text { Por Francia y por Alemania (Crónicas de la } \\
\text { Exposición) }\end{array}$ & 1890 & $\mathrm{BDH}$ \\
\hline Una Cristiana & 1890 & BVMC \\
\hline La Prueba & 1890 & RAG [18--?], BVMC \\
\hline Cuentos escogidos & 1891 & RAG \\
\hline Alarcón & ca. 1891 & $\mathrm{BDH}$ \\
\hline Polémicas y estudios literarios & 1891 & RAG, Gal., BDH \\
\hline La Piedra angular & 1891 & RAG, BDH \\
\hline Novelas ejemplares & 1891 & RAG [18-¿], Gal. [s.d.], BDH \\
\hline Nuevo Teatro Crítico & 1891-3 & Gal., BVMC \\
\hline Los franciscanos y Colón & 1892 & Gal. \\
\hline Cuentos de Marineda & 1892 & $\begin{array}{l}\text { RAG [18--?], Gal., BDH 1920, BVMC } \\
1964\end{array}$ \\
\hline
\end{tabular}




\begin{tabular}{|c|c|c|}
\hline Título de la obra & Año de edición & Versiones digitales \\
\hline Cuentos nuevos & 1894 & $\begin{array}{l}\text { RAG [18--?], Gal. [18--?], BDH 1910, } \\
\text { BVMC } 1964\end{array}$ \\
\hline Doña Milagros & 1894 & RAG [189-?], BVMC \\
\hline Los poetas épicos cristianos & 1894 & RAG [18--?], Gal. [18--?] \\
\hline Por la España pintoresca & 1895 & $\mathrm{BDH}$ \\
\hline Vida contemporánea: costumbres & 1896 & RAG [18--?], Gal., BDH \\
\hline Memorias de un solterón & 1896 & RAG [18--?], Gal. [18--?], BVMC \\
\hline Arco Iris: cuentos & са. 1896 & RAG [18--?], Gal. [18--?], BDH [18--?] \\
\hline Hombres y mujeres de antaño & $1896 ?$ & RAG, Gal., BDH \\
\hline El Tesoro de Gastón: novela & 1897 & RAG, Gal., BDH, BVMC \\
\hline Cuentos de amor & 1898 & BDH 1910-1915, BVMC 1920 \\
\hline El Vestido de boda & 1898 & BDH, BVMC 1906 \\
\hline El Saludo de las brujas & 1899 & RAG [18--?], Gal., BVMC \\
\hline $\begin{array}{l}\text { La España de ayer y la de hoy (conferencia } \\
\text { de París) }\end{array}$ & & RAG, Gal. \\
\hline El Niño de Guzmán & 1899 & BDH, BVMC 1900 \\
\hline Cuentos sacro-profanos & 1899 & $\begin{array}{l}\text { RAG [189-?], Gal. [189-?], BDH, BVMC } \\
1963\end{array}$ \\
\hline $\begin{array}{l}\text { Un Destripador de antaño (historias y } \\
\text { cuentos regionales) }\end{array}$ & 1900 & RAG [18--?], Gal. [18--?], BDH, BVMC \\
\hline En Tranvía. Cuentos dramáticos & 1901 & BDH 1899?) \\
\hline Cuarenta días en la exposición & 1901 & RAG [18--?], Gal. [18--?], BDH 1905 \\
\hline Por la Europa católica & 1902 & \\
\hline De siglo a siglo & 1902 & Bodleian Library \\
\hline Cuentos de Navidad y de Reyes & 1902 & \\
\hline $\begin{array}{l}\text { Los franciscanos y el descubrimiento de } \\
\text { América }\end{array}$ & 1902 & \\
\hline Misterio & 1902 & BVMC \\
\hline Sud-exprés & 1902 & RAG [19-i], Gal.[1920?], BVMC \\
\hline La Suerte, diálogo dramático & 1904 & BVMV 1906, BDH 1909 \\
\hline La Quimera & 1905 & RAG [18--?], Gal. [18--?], BVMC \\
\hline $\begin{array}{l}\text { Goya y la espontaneidad española: } \\
\text { conferencia... }\end{array}$ & 1905 & \\
\hline Discurso a la memoria de Gabriel y Galán & 1905 & \\
\hline Lecciones de literatura & 1906 & RAG [s.d.], Gal. [s.d.], BVMC 1910 \\
\hline Verdad: drama en cuatro actos & 1906 & BVMC \\
\hline
\end{tabular}




\begin{tabular}{|c|c|c|}
\hline Título de la obra & Año de edición & Versiones digitales \\
\hline Cuesta abajo & 1906 & BVMC, BHH 1909 \\
\hline El fondo del alma, cuentos del terruño & 1906 & BVMC \\
\hline Teatro & 1906 & BDH 1909, BVMC \\
\hline Cada uno: novela & 1907 & \\
\hline La Sirena negra & 1908 & BDH, BVMC 1981 \\
\hline Retratos y apuntes literarios & 1908 & \\
\hline Allende la verdad & 1908 & \\
\hline Finafrol: novela & 1909 & \\
\hline Cuentos de amor & [1910?] & BDH 1909?, BVMC 1912 \\
\hline La literatura francesa moderna & 1910- 1914 & BVMC \\
\hline Dulce dueño & 1911 & $\mathrm{BDH}, \mathrm{BVMC}$ \\
\hline En las cavernas & 1912 & \\
\hline Belcebú & 1912 & $\mathrm{BDH}$ \\
\hline Cocina española antigua & 1913 & \\
\hline Cocina española moderna & 1913 & \\
\hline Cuentos trágicos & 1914 & RAG [s.a.], Gal. [s.a.], BVMC 1963 \\
\hline Hernán Cortés y sus hazañas & 1914 & $\mathrm{BDH}$ \\
\hline Instinto & 1916 & BVMC \\
\hline La Ultima fada & 1916 & BVMC \\
\hline $\begin{array}{l}\text { Porvenir de la literatura después de la } \\
\text { guerra }\end{array}$ & 1917 & \\
\hline Francisco Pizarro & 1917 & \\
\hline Clavileño & 1917 & \\
\hline Los Tres arcos de Cirilo & 1918 & Gal. [s.a.], BDH, 1891 \\
\hline Bucólica & 1918 & RAG 1885, Gal. 1885 \\
\hline Dioses & 1919 & BVMC 1963 \\
\hline Rodando & 1920 & \\
\hline Cuentos de la tierra & 1922 & BDH, BVMC 1964 \\
\hline Cuadros religiosos & 1925 & $\mathrm{BDH}$ \\
\hline El Lirismo en la poesía francesa & 1926 & BVMC \\
\hline
\end{tabular}

